\title{
Oral dosing with papaya latex is an effective anthelmintic treatment for sheep infected with Haemonchus contortus
}

\author{
David J Buttle ${ }^{1}$, Jerzy M Behnke ${ }^{2 *}$, Yvonne Bartley ${ }^{3}$, Hany M Elsheikha ${ }^{4}$, David J Bartley ${ }^{3}$, Martin C Garnett ${ }^{5}$,
} Alison A Donnan ${ }^{3}$, Frank Jackson ${ }^{3}$, Ann Lowe $^{2}$, lan R Duce ${ }^{2}$

\begin{abstract}
Background: The cysteine proteinases in papaya latex have been shown to have potent anthelmintic properties in monogastric hosts such as rodents, pigs and humans, but this has not been demonstrated in ruminants.

Methods: In two experiments, sheep were infected concurrently with 5,000 infective larvae of Haemonchus contortus and 10,000 infective larvae of Trichostrongylus colubriformis and were then treated with the supernatant from a suspension of papaya latex from day 28 to day 32 post-infection. Faecal egg counts were monitored from a week before treatment until the end of the experiment and worm burdens were assessed on day 35 postinfection.

Results: We found that the soluble fraction of papaya latex had a potent in vivo effect on the abomasal nematode $H$. contortus, but not on the small intestinal nematode T. colubriformis. This effect was dose-dependent and at tolerated levels of gavage with papaya latex $(117 \mu \mathrm{mol}$ of active papaya latex supernatant for 4 days), the $\mathrm{H}$. contortus worm burdens were reduced by $98 \%$. Repeated treatment, daily for 4 days, was more effective than a single dose, but efficacy was not enhanced by concurrent treatment with the antacid cimetidine.

Conclusions: Our results provide support for the idea that cysteine proteinases derived from papaya latex may be developed into novel anthelmintics for the treatment of lumenal stages of gastro-intestinal nematode infections in sheep, particularly those parasitizing the abomasum.
\end{abstract}

\section{Background}

The traditionally available broad-spectrum anthelmintics for treatment of gastro-intestinal nematode infections in ruminants are largely limited commercially to three classes of drugs, namely benzimidazoles, imidazothiazoles/tetrahydropyrimidines, and macrocyclic lactones, which have been in use extensively for some 30 to 50 years. New classes have been launched in the last few years [1,2], represented by emodepside, monepantel, and derquantel, but these drugs are fairly expensive and so far have very limited label claims for efficacy. For example, emodepside is labelled only for treatment of enteric worms in cats, and in combination with praziquantel

\footnotetext{
* Correspondence: jerzy.behnke@nottingham.ac.uk

${ }^{2}$ School of Biology, University of Nottingham, University Park, Nottingham, NG7 2RD, UK

Full list of author information is available at the end of the article
}

tablets (Profender ${ }^{\circledR}$ ) for dogs, and monepantel and derquantel (in combination with abamectin) are labelled only for use in sheep. Nonetheless, while it is likely that some of these drugs may be labelled for use in more species in the future, their cost will be much higher than products of the older anthelmintic classes. Thus, it appears most likely that the development of resistance will continue to outpace the introduction of new anthelmintic drugs, and any new drugs will be much more expensive. As a result, there is a need for more therapeutic agents in the market, because of the rapidity with which resistance develops in nematodes when anthelmintics are applied intensively under typical large flock industrial-scale husbandry conditions $[3,4]$.

Natural plant-derived products have been known for many decades to possess anthelmintic properties $[5,6]$ and yet generally these have been inadequately

\section{() Biomed Central}


researched and none have been taken to the market stage. One such group of potential anthelmintics is the cysteine proteinases found in fruits such as papaya, figs, kiwi fruits and pineapples. Laboratory experiments have demonstrated clearly that these compounds damage intestinal nematodes of rodents by a novel mechanism, targeting the cuticle which at first blisters, and then is disrupted and weakened sufficiently to enable the internal hydrostatic pressure to rupture the body wall and result in the disintegration of the worms [7]. Papaya latex, which contains high concentrations of four distinct cysteine proteinases [8], has been shown to have potent activity against worms of the stomach, small and large intestine in rodent model systems in vivo, causing dosedependent reductions of worm burdens within safe treatment margins that resulted in little or no pathology [9-11]. Fig and papaya extracts have also been shown to reduce intestinal helminth infections in humans and in other monogastric animals including pigs [12,13].

Ruminants pose a formidable challenge for anthelmintic therapy, because after oral drenching the drugs pass first into the rumen, where their concentrations are greatly diluted and where they may reside for some time before gaining access to the abomasum and remaining sections of the intestinal tract [14]. Moreover the microbial and protozoal fauna of the rumen can cause drug efficacy to deteriorate. Indeed, papaya latex has previously been reported to have no efficacy against Haemonchus contortus in sheep [15] and to be highly toxic in ruminants [16].

Nevertheless, since papaya latex clearly has anthelmintic activity in monogastric animals, we were interested in reassessing the efficacy of papaya latex in sheep infected with nematodes. $H$. contortus was selected, because economically this is perhaps the most important parasite of sheep globally, responsible for major losses to the sheep industry [17]. We also assessed efficacy against Trichostrongylus colubriformis, which resides in the mucosa of the small intestine and which therefore is less exposed to compounds passing through the gut. In this paper we report the results of two experiments conducted in groups of lambs sufficient for robust statistical evaluation. These studies were aimed at determining the dose-dependence of four treatments at 24-hour intervals, the efficacy of a single dose, and the impact of neutralising abomasal acidity on the efficacy of papaya latex.

\section{Materials and methods}

Animals

In both experiments Greyface crosses [blueface Leicester tups on Scottish blackface ewes] were used. They were bred, raised and housed under conditions to minimise parasite contamination at the Moredun Research Institute animal holding facility. Each animal was screened for the presence of worms by faecal egg count examination prior to the start of the experimental infection. The sheep were approximately 4-5 months-old at the time that they were infected.

\section{Parasites}

The anthelmintic susceptible isolates of $H$. contortus (MHco3; [18]) and T. colubriformis (MTco1; unpublished data) were used in these trials.

\section{Papaya latex}

Four kg of Carica papaya spray-dried latex (Enzymase, P1) were dissolved in 12 litres of water. The preparation was centrifuged at $17,700 \times g$ and the pellet was discarded. The supernatant was concentrated to a third of its original volume by placing in dialysis tubing [MW cut-off 3,500 (SpectraPor $45 \mathrm{~mm}$ diam.)] over polyethylene glycol 20,000. The concentrated material was aliquoted into individual containers and freeze-dried ready for reconstitution and oral delivery. Throughout the paper we refer to this preparation as the papaya latex supernatant (PLS).

At each step of the purification the molar concentration of active cysteine proteinase was assessed by titration with L-trans-epoxysuccinyl-leucylamido (4-guanidino)- butane (E64, Sigma-Aldrich) [19] with $4 \mathrm{mM}$ L-cysteine as a reducing agent. The starting amount was $70 \mathrm{mmols}$ of active enzyme in $4 \mathrm{~kg}$ of spray-dried latex, and during purification $41 \mathrm{mmols}$ were retained in the freeze-dried supernatant. The individual aliquots of freeze-dried preparation contained 47, 117 and $234 \mu \mathrm{mol}$ active cysteine proteinase by E-64 titration, these being the individual doses used for Experiment 1 (below). Throughout, once aliquoted and frozen, there was no deterioration of the enzyme activity and values derived before and after experiments were very similar.

\section{Haematocrit measurements}

Venous blood was collected by jugular venepuncture into $10 \mathrm{ml}$ heparinised vacutainer tubes (Becton Dickinson vacutainers systems) for microhaematocrit analysis expressed as percentage packed cell volume (PCV).

\section{Faecal egg counts}

Faecal samples were taken per rectum throughout the course of the trial and faecal egg counts (FEC) were conducted on the material using a modification of the technique described by Jackson [20]. Total faecal egg counts were differentiated into Haemonchus and Trichostrongylus using previously described methodologies $[13,14]$. In brief the eggs from one gram of faeces from all individuals in each group were pooled and stained with peanut agglutinin (PNA) lectin. The PNA lectin 
binds preferentially to the carbohydrates on the egg shell of $H$. contortus [21,22].

\section{Necropsy and worm counts}

All of the animals were necropsied on day 35 post infection (p.i.) using the post mortem and worm recovery methods previously described [23]. The total worm burdens from the abomasa and small intestines were estimated from a sub-sample of the washings and saline digests. Ten percent subsamples $(500 \mathrm{ml})$ were taken and stored.

Two percent aliquots were examined at $\mathrm{x} 40$ using a stereo microscope. The data from the washings and saline digests were pooled to provide total worm burden data. Recovered worms were sexed, staged and speciated [24].

\section{Cimetidine treatment}

The antacid cimetidine (from the Pharmacy at the Queen's Medical Centre, Nottingham) (3.2 g in $50 \mathrm{ml}$ water) was administered 20 mins before PLS in animals that received both treatments.

\section{Ethical approval}

All experimental procedures described in this manuscript were examined and approved by the Moredun Research Institute Experiments and Ethics Committee and were conducted under approved British Home Office licenses in accordance with the Animals (Scientific Procedures) Act of 1986.

\section{Experimental design}

Two experiments were conducted. The first experiment was designed to determine the efficacy of different amounts of PLS administered on 4 consecutive days on $H$. contortus and T. colubriformis faecal egg counts and worm burdens (dose-response experiment). The second experiment was split into three different sections. The first of these was to confirm any dose-dependent effect of PLS, the second investigated the efficacy of a single dose compared to 4 repeat doses of PLS, and the third examined any possible effect of an antacid, cimetidine, and additive or synergistic effects of the co-administration of cimetidine, with PLS.

For both Experiments $1(n=20)$ and $2(n=30)$ lambs were each infected with 5,000 infective larvae (L3) of $H$. contortus and 10,000 T. colubriformis $\mathrm{L}_{3}$ on day 0 . The animals were randomly separated into groups of five. Treatments started on day 28 p.i., and for 3 consecutive days thereafter, aliquots of PLS preparations and cimetidine, as appropriate, were reconstituted with $50 \mathrm{ml}$ of water and delivered orally by gavage. Animals were euthanased on day 35 p.i. unless otherwise stated.

\section{Experiment 1}

The following treatment groups comprised Experiment 1: Group 1 - water only, once daily for four days

Group 2 - $47 \mu \mathrm{mol}$ active cysteine proteinase, once daily for four days

Group 3 - $117 \mu \mathrm{mol}$ active cysteine proteinase, once daily for four days

Group $4-234 \mu \mathrm{mol}$ active cysteine proteinase, once daily for three days

Note: Group 4 animals only had 3 treatments and were slaughtered on day 32 p.i.

FEC were conducted on days 21, 23, 25, 28, 29, 30, 31, 32 and 35 p.i. and on each occasion, except day 32, eggs were differentiated as described above.

\section{Experiment 2}

This experiment comprised the following groups:

Group 1 - water only, once daily for four days

Group 2 - $50 \mu \mathrm{mol}$ active cysteine proteinase, once daily for four days

Group 3 - $100 \mu \mathrm{mol}$ active cysteine proteinase, once daily for four days

Group 4 - $100 \mu \mathrm{mol}$ active cysteine proteinase, single treatment

Group 5 - cimetidine $3.2 \mathrm{~g}$, once daily for four days

Group 6 - cimetidine $3.2 \mathrm{~g}$ then $50 \mu \mathrm{mol}$ active cysteine proteinase, once daily for four days

FEC were conducted and eggs were differentiated as described above.

\section{Pathology}

Immediately after euthanasia, all the carcasses were examined for any gross lesion and samples from the brain (internal capsule, hippocampus and midbrain) liver, kidney, lung and rumen were collected and placed into $10 \%$ formal saline. After fixation for five days, the samples were processed for histopathology by standard procedures for Haematoxylin and Eosin staining [25].

\section{Statistical analysis}

Data are presented as arithmetic means and also as Log means [for FEC these were $\log _{10}(E P G+25)$ and for worm counts $\log _{10}($ total worms +10$\left.)\right]$ because FEC and worm burdens are typically overdispersed in animals, and these transformations have been found in the past to best stabilise variance. Changes over time in FEC were carried out on $\log _{10}(E P G+25)$ transformed data and analysed by repeated measures GLM (rmGLM) in SPSS (version 16.0.0 for Windows). If the assumptions of sphericity were not met, as assessed by Mauchley's test, we used the Huynh-Feldt adjustment to the degrees of freedom to err on the side of caution.

Worm counts were analysed by non-parametric tests, the Mann-Whitney $U$ test for 2 group comparisons, the 
Kruskal-Wallis test for larger group treatment effects, and correlational relationships were examined with Spearman's rank order test, all using the software package SPSS (version 16.0.0 for Windows). For testing specific hypotheses we used the non-parametric Jonckhere-Terpstra test and for analyzing a $2 \times 2$ design the 2-way non-parametric ANOVA, both in the software package "Asking Questions in Biology", based on Barnard et al [26] and on Meddis [27].

\section{Results}

Experiment 1 - Dose-response effects of papaya latex supernatant on $\mathrm{H}$. contortus and $T$. colubriformis

In the control group treated with water, mean undifferentiated FEC values (Figure 1) were fairly steady throughout the period of observation, with arithmetic means varying from $6349( \pm 1182$; standard error of the mean) on day 21 p.i. to $12010( \pm 2247)$ on day 35 p.i. with a peak count of $14143( \pm 2875)$ on day 31 p.i. As can be seen from Figure 1, undifferentiated FEC in all three groups treated with PLS showed some reduction in the days after treatment in contrast to the watertreated control group which rose marginally. In relation to day 28, just prior to treatment, and based on arithmetic means, FEC rose in the control group on day 35 by $12.0 \%$, fell in Group 2 (47 $\mu \mathrm{mol}$ of PLS) by $66.4 \%$ and by $74.8 \%$ in Group 3 (117 $\mu$ mol of PLS). In Group 4 ( $234 \mu \mathrm{mol}$ of PLS) FEC fell on day 32 by $79.4 \%$.

Group 4 (receiving the highest dose of PLS) was culled earlier on day 32 p.i. so that statistical analysis of FEC was based only on the first three groups, and this indicated that there was a significant main effect of treatment (between subjects effect, $F_{2,10}=7.95, p=0.009$ ). There was also a significant divergence between the groups in FEC over time (within subjects test, 2-way interaction time " treatment, $F_{13.8,69.0}=7.43, p<0.001$ ).

As expected, differential FEC showed that the majority of the eggs were from $H$. contortus, and that it was this species that was primarily affected by treatment (Figure 2A; note that no differential egg counts were conducted on day 32). As in the total FEC, there was a marked drop after treatment that was dose-related, and in the groups given the highest treatments, there was no subsequent rise between day 31 (last day of treatment) and autopsy on day 35.

The number of $T$. colubriformis eggs in faeces dropped during the treatment period, but then picked up soon afterwards in most groups (Figure 2C), increasing by d 35 even in the animals treated with $117 \mu \mathrm{mol}$ of active enzyme. However, in group 4, treated with $234 \mu \mathrm{mol}$, FEC were still low on d31 compared to earlier days, a day before these animals were culled.

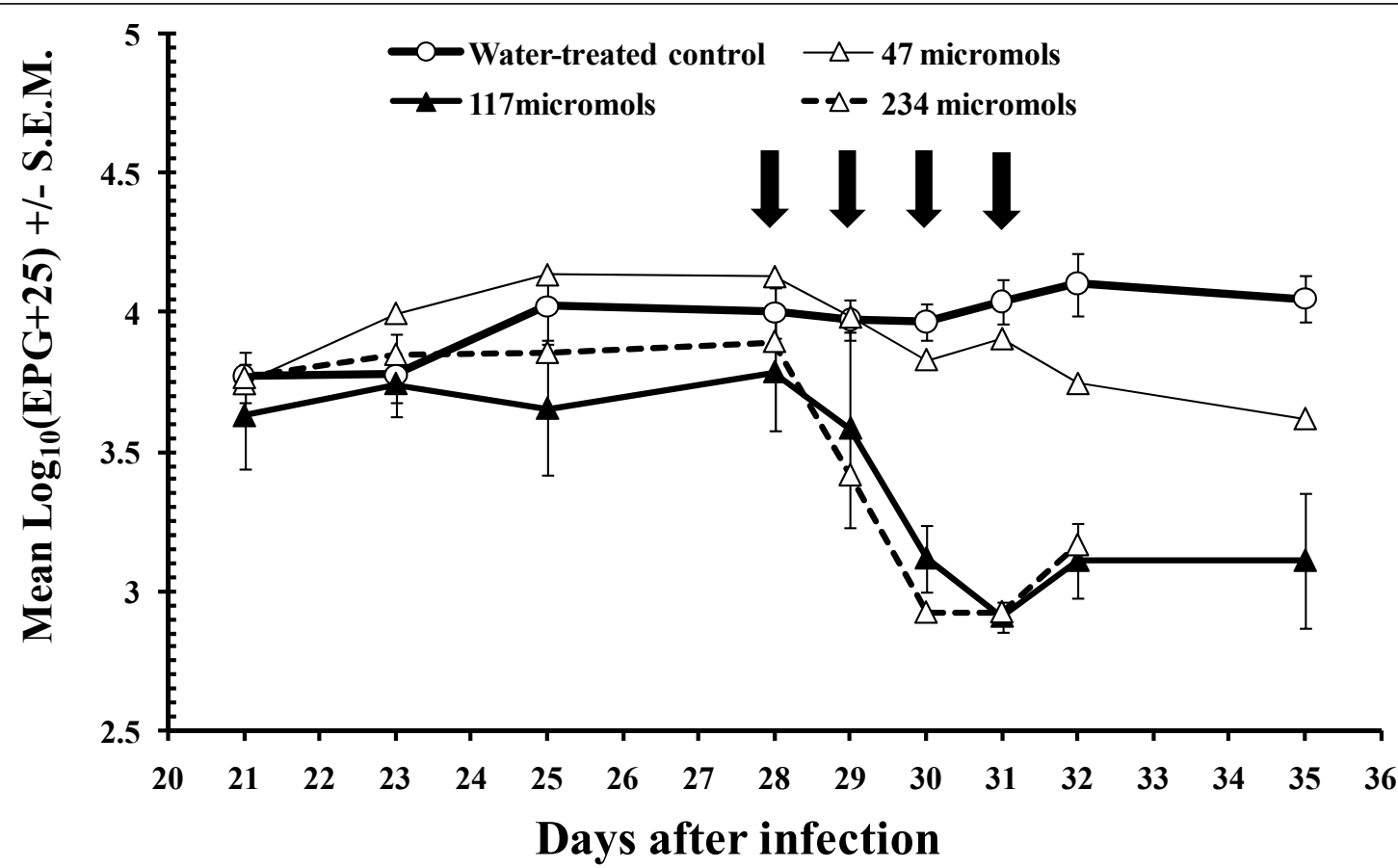

Figure 1 Faecal egg counts during the course of Experiment 1. Mean group values based on log 10 (EPG +25$)$ transformed data ( \pm S.E.M.) with 5 animals per group. The bold vertical arrows show the days on which treatment was administered, the first dose being given on day 28 p.i. 


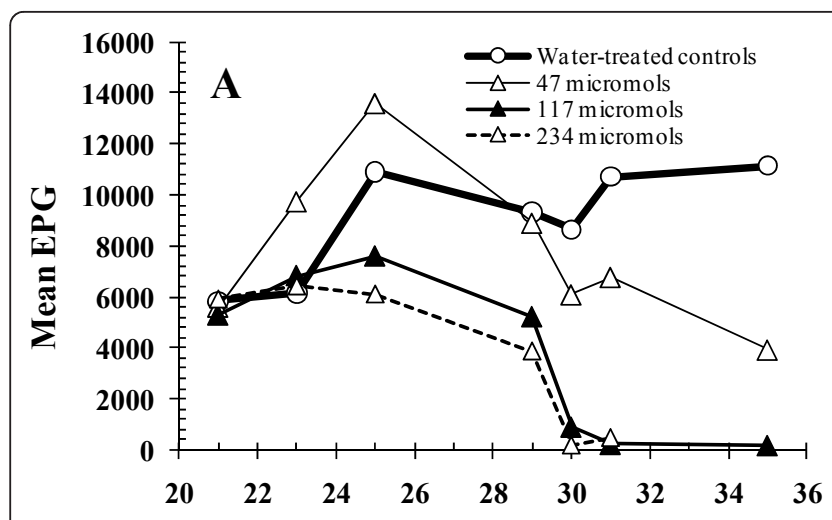

Days after infection

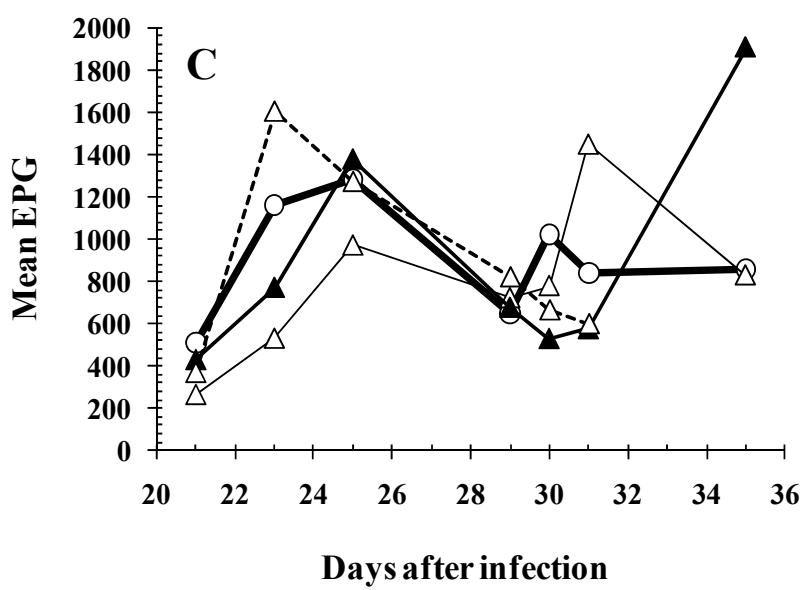

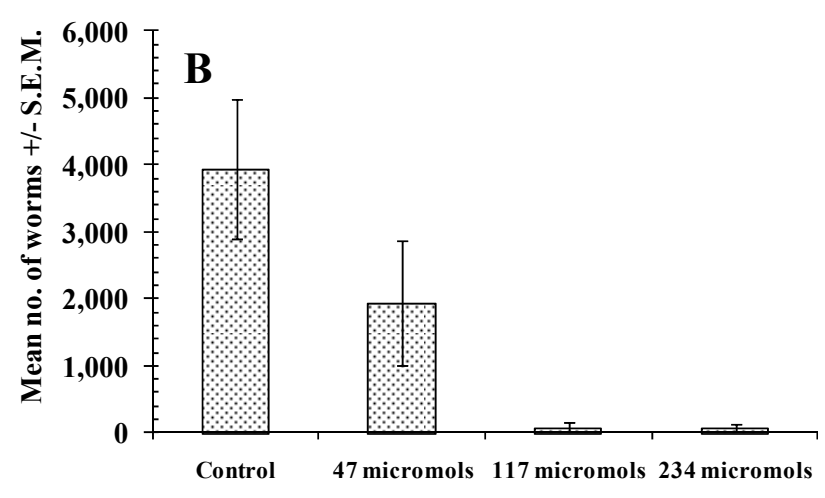

Treatment

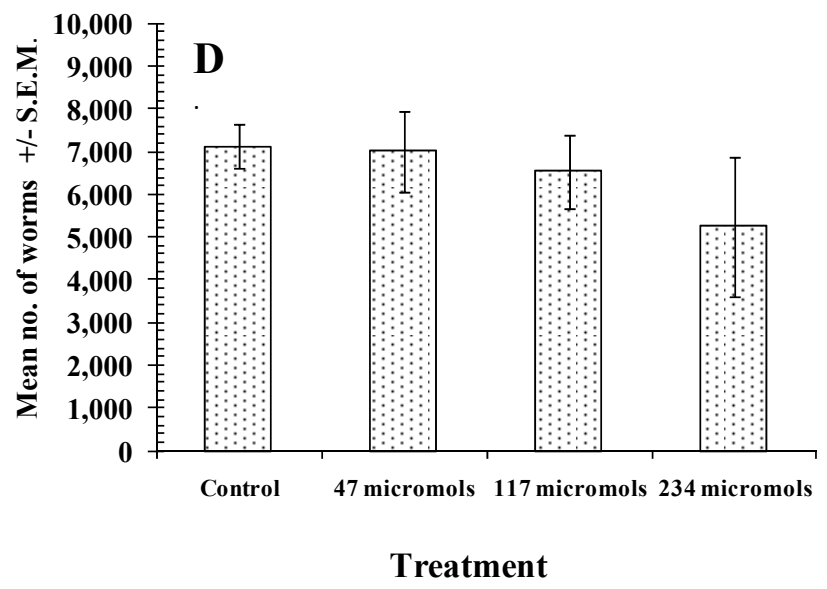

Figure 2 Faecal egg counts (FEC) and worm burdens for $\boldsymbol{H}$. contortus and $\boldsymbol{T}$. colubriformis in Experiment 1 . A, FEC attributable to H. contortus, based on differential counts on pooled faeces at each time point, except day 32 p.i., when differential egg counts were not conducted; B, H. contortus worm burdens; C, FEC attributable to T. colubriformis based on differential counts, as above; D, T. colubriformis worm burdens. For details of treatments, differential counts and statistical analysis see text.

In line with the drop in FEC with treatment, the two highest doses of PLS cleared $H$. contortus worms almost entirely from the abomasa (Figure 2B). Treatment with $117 \mu \mathrm{mol}$ and $47 \mu \mathrm{mol}$ resulted in $98.1 \%$ and $51.0 \%$ reduction in worm burdens, respectively. There was a highly significant effect of treatment (Kruskal-Wallis test, $\chi_{3}^{2}=11.57, P=0.009$ across all 4 treatment groups), that was dose-related $\left(r_{s}=-0.773, n=19, p<0.001\right)$.

There was no significant effect of treatment on T. colubriformis worm counts (Figure 2D) (KruskalWallis test, $\chi^{2}{ }_{3}=0.55, p=\mathrm{NS}$ across all 4 groups). However, the data in Figure 2D suggest that T. colubriformis worm counts may have been lower in the group receiving the highest dose of PLS (log mean 26.1\% lower relative to the control group) but comparison of the worm counts in this group (Mann-Whitney $U$ test) with the water-treated control group gave $z=-0.522$, and $p=$ NS. The lower mean count was attributable to two animals that had low counts (600 and 2200 worms), below the control range (5600-8700), the other 3 being well within and even above the control range (6950-9100).

\section{Haematocrits}

Table 1 shows that PCV were highest in the animals treated with the highest doses of PLS, with a significant difference between treatment groups $\left(\chi_{3}^{2}=9.4, p=\right.$ 0.025). This suggests a dose-response curative effect on haemonchosis-induced anaemia in the animals.

Table 1 Arithmetic mean packed cell volume ( \pm SEM) of sheep in treatment groups on day 31 post infection

\begin{tabular}{cc}
\hline Treatment & Packed cell volume (SEM) \\
\hline Water-treated control & $21.5(2.4) n=5$ \\
$47 \mu \mathrm{mol}$ active proteinase & $20.3(1.6) n=5$ \\
$117 \mu \mathrm{mol}$ active proteinase & $27.9(1.2) n=4$ \\
$234 \mu \mathrm{mol}$ active proteinase & $27.4(1.7) n=5$ \\
\hline
\end{tabular}




\section{Pathological examination}

No gross lesions were appreciated in any of the sheep during the post-mortem studies.

Histologically, two animals showed mild focal interstitial inflammatory mononuclear infiltrates in liver, lung or rumen. There was a focal infiltration of a few mononuclear cells in the alveolar wall of one animal (4636G; $234 \mu \mathrm{mol}$ group). Only one sheep (4634G; $234 \mu \mathrm{mol}$ group) showed lesion in more than one organ: focal mild infiltration of mononuclear cells in the lamina propria of the ruminal papillae and mild hypercullarity of portal spaces in the liver.

\section{Experiment 2}

Dose-dependence of the effect of PLS given over 4 days

As Figure 3A shows, treatment with PLS resulted in an immediate fall in FEC, and this reduction was more marked in the sheep receiving the higher dose (100 $\mu \mathrm{mol}$ active enzyme). The divergence between treatment groups was significant [rmGLM on $\log _{10}$ (EPG $+25)$ transformed data, within subjects test, interaction between time and treatment, $F_{5.4,32.5}=3.63, p=0.009$ ] There was a significant negative correlation between dose of active enzyme and EPGs on d35, the final day of the experiment $\left(r_{s}=-0.66, n=15, p=0.007\right)$.

Based on the highly significant dose-response found in Experiment 1, the hypothesis that $H$. contortus worm counts should be highest in the water-treated control group (group 1), intermediate in the animals given $50 \mu \mathrm{mol}$ of PLS (group 2) and lowest in those treated with $100 \mu \mathrm{mol}$ (group 3), was tested. The results are illustrated in Figure 3B, where it can be seen that worm counts declined with increasing dose of treatment [nonparametric Jonckhere-Terpstra test $z=2.02$ ( $p=$ $0.0218)$ ]. Based on arithmetic mean worm burdens, and compared to the water-treated control group (4130 \pm 1030 ) worm burdens fell by $43.6 \%$ in the group treated with $50 \mu \mathrm{mol}$ of PLS for 4 days $(2330 \pm 832)$ and by $79.2 \%(860 \pm 355)$ in those given $100 \mu \mathrm{mol}$. There was a significant negative correlation between worm counts and increasing doses of PLS (Spearman's one-tailed test $\left.r_{s}=-0.539, p=0.019\right)$. As in Experiment 1, and as illustrated in Figure 3C, T. colubriformis worm burdens did not decline with increasing dose of PLS. These results confirm the findings of Experiment 1, namely that treatment with PLS reduced $H$. contortus worm burdens in a dose-dependent manner but was without effect on T. colubriformis.

Comparison of repeated administration of PLS with a single dose

$100 \mu \mathrm{mol}$ of active cysteine proteinase administered as a single dose was compared with the same dose administered on 4 consecutive days and with a water control. Analysis of egg counts by rmGLM [duration of PLS treatment*time, on $\log _{10}(E P G+25)$ transformed data, within subjects test] gave $F_{6.0,35.9}=5.03(p=0.001)$ when the water-treated control group was included, indicating that FEC had diverged significantly with time between the 3 groups (Figure 4A). However, confining the analysis to the two infected groups to ascertain whether repeated administration was more effective than a single dose treatment gave only a borderline significance (treatment*time interaction, $F_{2.4,19.3}=3.21$, $p=0.053)$ and there was no significant difference between the single dose treatment and the control group (post-hoc test, treatment*time interaction, $F_{1,8}$, = $1.26, p=0.3)$. The main effect of treatment was not significant in any of these combinations.

Sheep that had received 4 doses of PLS had fewer $H$. contortus worms compared to those that had received the single treatment (2-tailed Mann-Whitney $U$ test, $z=$ -2.4, $p=0.016$ ) (Figure 4B). However, there was no evidence for a reduction in worm burden with a single treatment. The animals given a single dose of $100 \mu \mathrm{mol}$ of PLS had a worm burden with a higher arithmetic mean $(4080 \pm 716$ worms) than the water-treated controls $(3500 \pm 1335)$ (Figure $4 \mathrm{~B})$. In respect of T. colubriformis worm burdens, the group given a single dose of PLS (group 4) had a lower arithmetic mean (4590 \pm 429 ) than the water-treated control group (group 1; $5070 \pm 106$ ) and the repeatedly treated group (group 3; $5020 \pm 375)$, but there was no significant difference between treated groups (Mann Whitney $U$ test, group 3 vs group 4, $z=-0.73, p=\mathrm{NS}$ ).

\section{Effect of co-administration of an antacid and PLS}

The co-administration of an antacid, cimetidine, with papaya latex has been shown to greatly increase efficacy against a murine stomach nematode [9], presumably because of the weak activity of the papaya proteinases at $\mathrm{pH}$ values $\leq 4.0[28,29]$. It has also been reported that cimetidine had an independent anthelmintic effect when administered directly into the abomasum [30]. We therefore decided to test the effect of cimetidine when administered orally, and investigate any additive or synergistic effect of cimetidine co-administered with a sub-optimal dose of PLS (50 $\mu \mathrm{mol}$ active cysteine proteinase). The FEC data are shown in Figure 5A. The summary data suggest that treatment with $50 \mu \mathrm{mol}$ of active enzyme caused a rapid, sustained drop in FEC, relative to the control group. However, analysis by 2 -way rmGLM with PLS treatment and cimetidine treatment, as factors and time as the within subjects factor, indicated that the divergence of FEC between treatment groups was of borderline significance only [PLS treatment "time, on $\log _{10}$ $(E P G+25)$ transformed data, within subjects test, $F_{3.5}, 56.4$ $=2.589, p=0.053]$. There were no other significant terms in the full factorial model, and post hoc tests on individual days after the start of treatment did not 

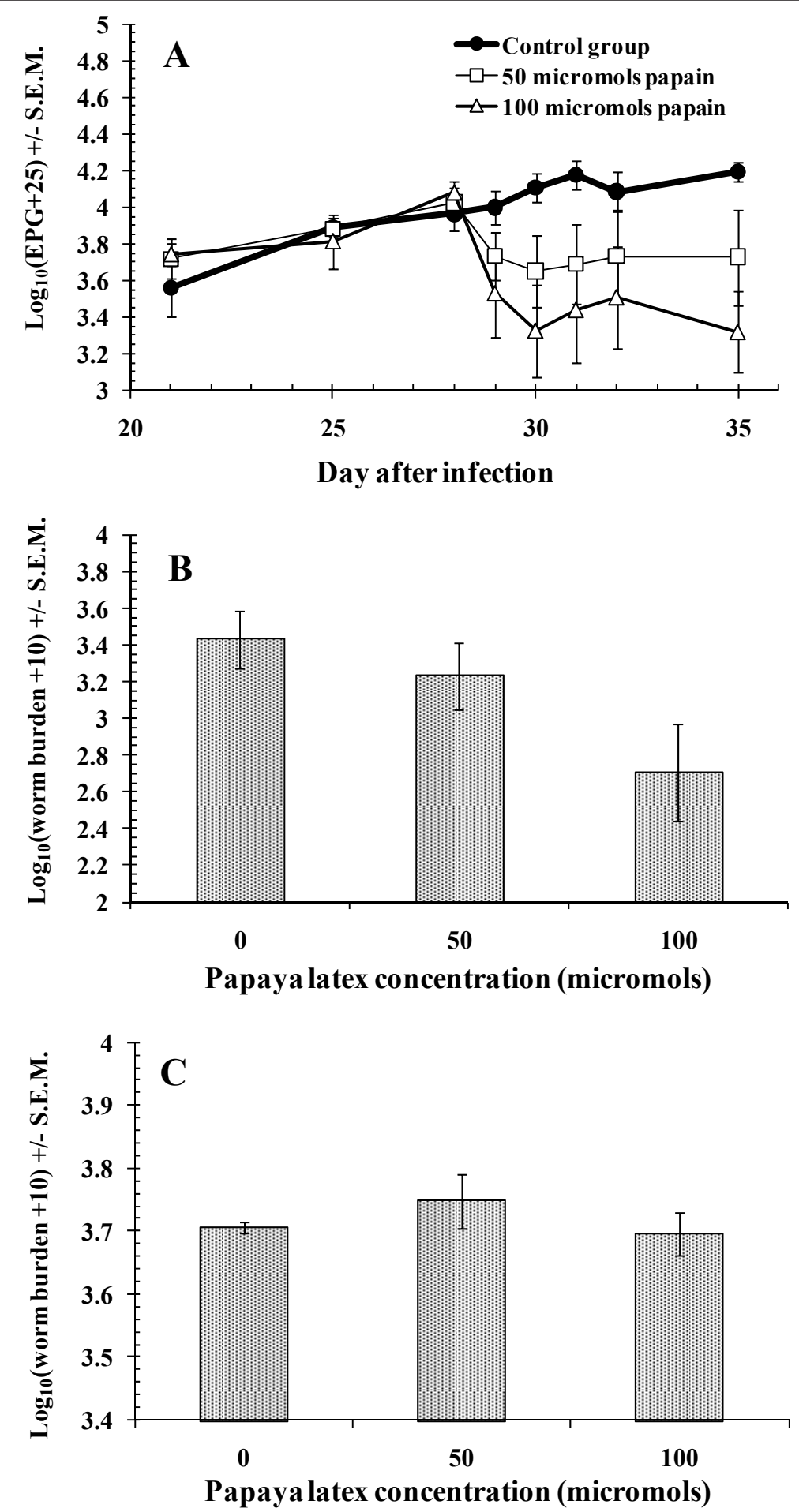

Figure 3 Total FEC and individual worm burdens in sheep treated with different doses of PLS in Experiment 2. A, Total FEC. The data are mean group values based on $\log _{10}(E P G+25)$ transformed data $( \pm$ S.E.M.) with 5 animals per group. Repeated treatment was administered, the first dose being given on day 28 p.i. and the last on day 31; B, H. contortus worm burdens; C, T. colubriformis worm burdens. 

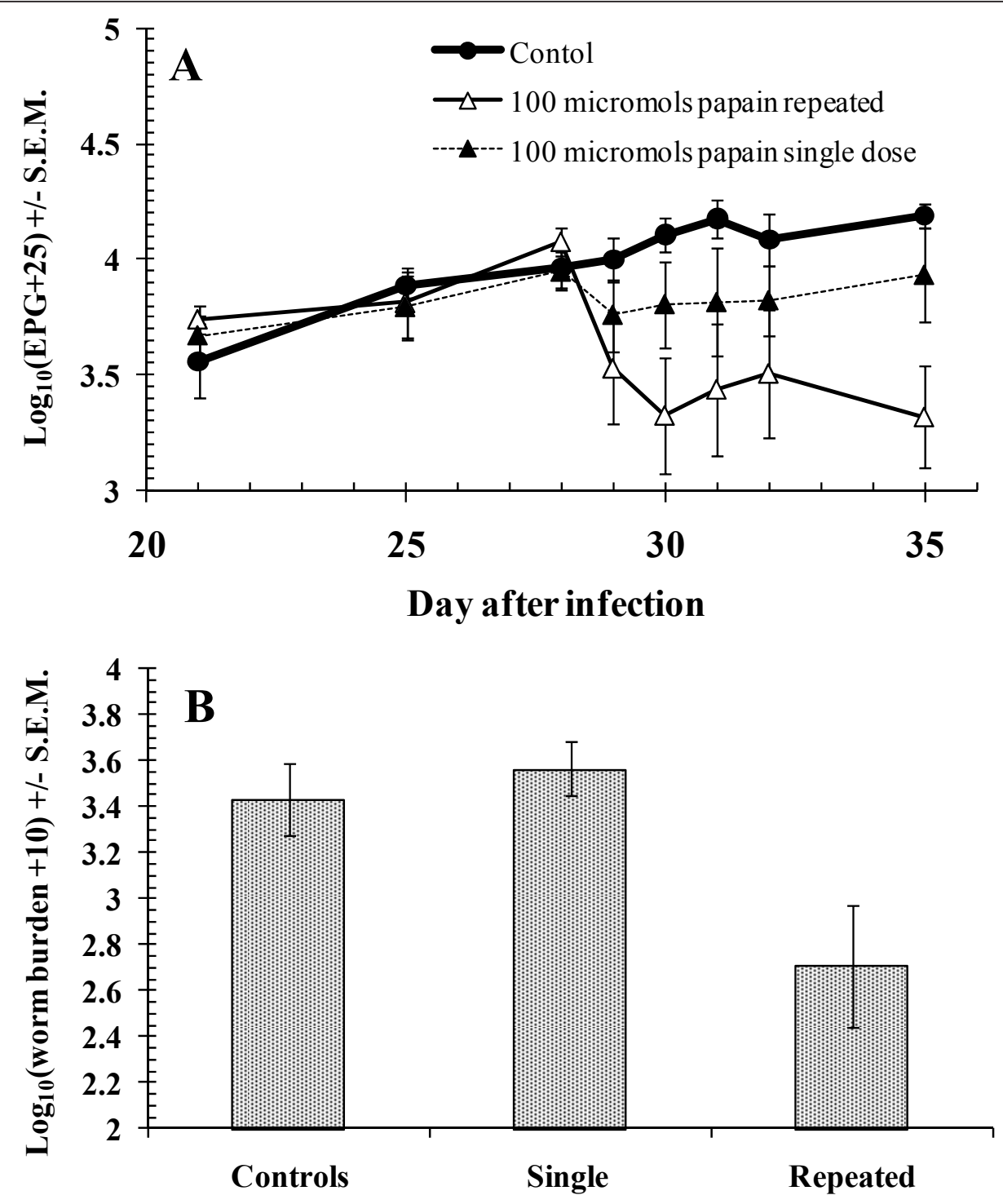

Treatment

Figure 4 Comparison of a single dose of $100 \mu \mathrm{mol}$ PLS with 4 daily doses. A, Total faecal egg counts; $\mathbf{B}$, H. contortus worm burdens.

provide support for any significant differences between treatment groups. The analysis therefore indicated that there was no enhancement of the anthelmintic activity when PLS was administered with the antacid cimetidine, and no independent anthelmintic effect of cimetidine when administered orally alone.

The results of worm counts of $H$. contortus are shown in Figure $5 \mathrm{~B}$. The data were analyzed by a 2 -way nonparametric ANOVA. The two groups treated with PLS had marginally lower mean worm burdens (arithmetic means for group $2=2330( \pm 833)$ and group $6=2530$ $( \pm 954)$, compared with group $1=3500( \pm 1335)$ and group $5=4130( \pm 1030)$, but at this dose of enzyme activity $(50 \mu \mathrm{mol})$, there was no significant reduction in worm burdens, although this was just outside the cutoff $(z=1.36, p=0.087)$. There was no enhancement of the anthelmintic effect in the animals co-administered cimetidine and PLS, and there was no overall effect of cimetidine on worm burdens.

For T. colubriformis there was no effect of PLS or of cimetidine, given separately or in combination (worm burdens in control group $1=5070 \pm 10.6$, and in group 2 treated with $50 \mu \mathrm{mol}=5700 \pm 596$; the cimetidinetreated group 5 had $5040 \pm 358$ worms and that given both $50 \mu \mathrm{mol}$ of PLS and cimetidine (group 6) had $5270 \pm 362$ worms). 

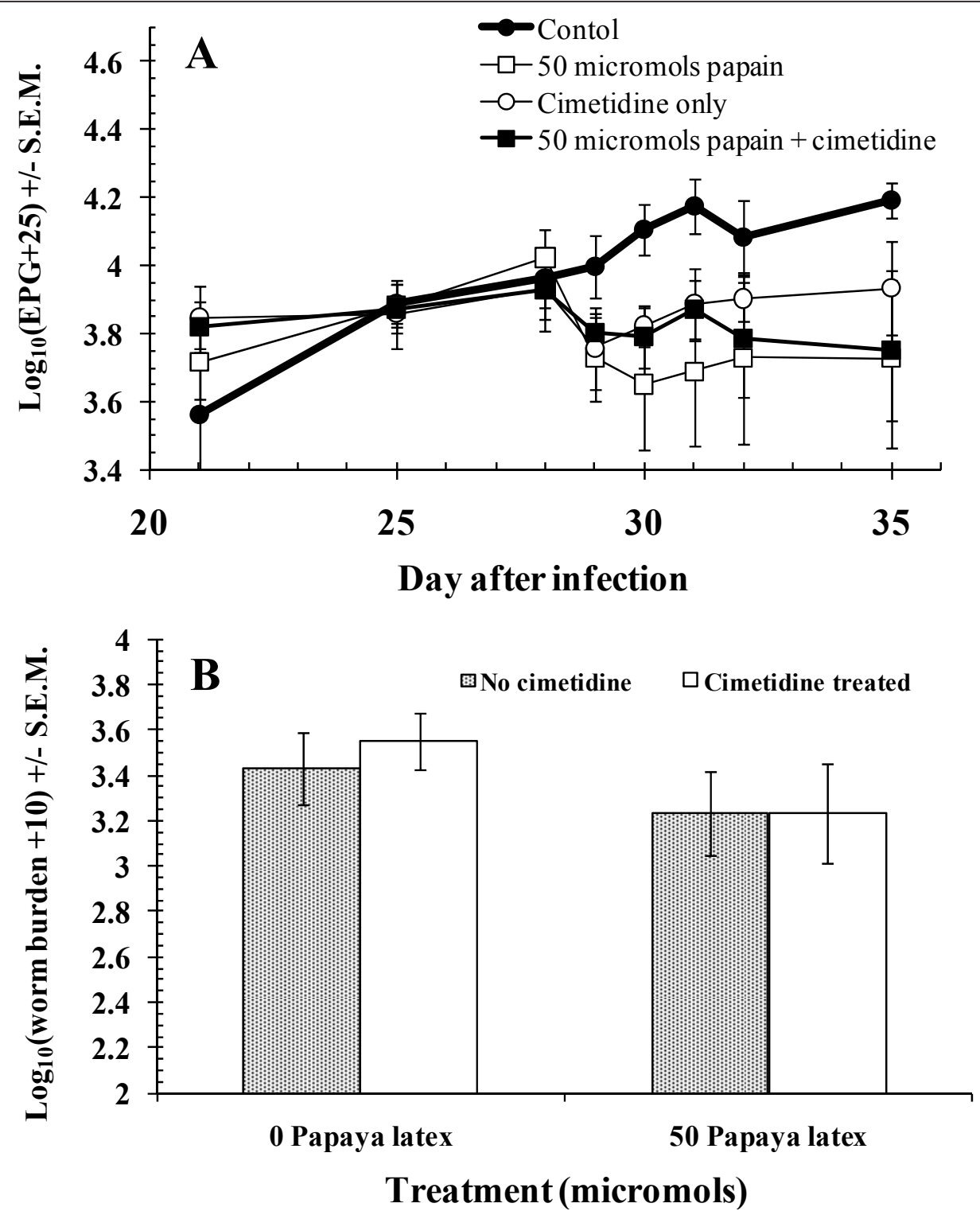

Figure 5 Effect of cimetidine and PLS, administered separately or in combination, on total faecal egg counts and $\mathrm{H}$. contortus worm burdens. A, Total FEC; B, H. contortus worm burdens. Cimetidine $(3.2 \mathrm{~g})$ and PLS (50 $\mu \mathrm{mol})$ were administered in isolation or in combination, on 4 consecutive days. In the latter case cimetidine was administered 20 min before PLS.

\section{Discussion}

The results of the present study demonstrate for the first time that PLS possesses potent anthelmintic activity capable of clearing the adult parasitic nematode $H$. contortus from the sheep abomasum. The lack of efficacy of a single dose compared with the use of 4 daily doses suggests that, following dilution in the rumen, the enzymes require prolonged contact time with the worms in order to prove effective. Our results contrast with an earlier report of high toxicity of papaya latex in ruminants [16]. Although two animals showed histological lesions, they were mild and scant, and therefore considered not significant or related to diets or treatments administered to the animals. The presence of mild inflammatory infiltrates in the digestive system, liver or lungs is common in ruminants reared in outdoors or semi-outdoor conditions and we therefore consider them to be non-specific.

The in vivo anthelmintic efficacy of crude papaya latex against murine nematodes residing in various parts of the GI tract, has been reported previously [9-11]. However, in order to be able to scale up sufficiently to treat ruminants it was necessary to find a way to concentrate the active principles, the papaya cysteine proteinases 
found in the latex. A simple procedure of separation and concentration of the supernatant fraction allowed this, and at the same time may have removed contaminants that are toxic in ruminants. In our experiments, no toxic effects were detected with crude latex at effective anthelmintic doses in mice [11] nor was there any sign of toxicity in a pilot experiment where sheep were treated with crude latex preparations by gavage (results not shown). It is possible to monitor accurately the molar amount of active cysteine proteinase by the use of an active-site titrant, E64, that binds irreversibly to the active site of the enzymes and blocks activity, on a 1:1 molar basis $[19,31]$. The animals were dosed on this basis, rather than on the weight of the crude latex. Enzyme activity in papaya latex varies depending on the method of its preparation as well as other factors, and a highly active spray-dried preparation that is available in bulk and is employed in many manufacturing processes http://www.enzymase.com/production/process.htm was used as our starting material.

In our experiments, the effect of PLS was dose-dependent, consistent between experiments and enhanced by repeated dosing over 4 days, but not by co-treating with antacid (unlike earlier observations using murine stomach nematodes [9]). One reason for the lack of effect of an antacid may be the reported impact of nematode infection on abomasal $\mathrm{pH}$. Infection with Teladorsagia (Ostertagia) circumcincta or $H$. contortus may lead to a rise in abomasal $\mathrm{pH}$ from as low as $\mathrm{pH} 2.5$ up to approximately 6.5-7.0 [32,33], which is close to the $\mathrm{pH}$ optimum of the papaya cysteine proteinases, and therefore there was little scope for cimetidine to alter $\mathrm{pH}$ further. In our experiments abomasal $\mathrm{pH}$ was not monitored because this would have necessitated an abomasal fistula for the purpose and ethical approval was not granted. The increase in abomasal $\mathrm{pH}$ in infected animals suggests that the enzymes would only remain active in the abomasa in the presence of an infection, and that in animals with no or low worm burdens the abomasal pH would inhibit enzyme activity, thus preventing any unwanted side-effects on healthy animals.

The location of $T$. colubriformis in the mucosa, rather than the lumen, of the small intestine may explain its resistance in vivo. It is relevant that other species of intestinal nematodes that have temporary developmental stages in the tissues of their hosts are not affected by plant cysteine proteinases during the phases of their life cycles when they are actually in the mucosa as opposed to the intestinal lumen [10]. Given that we found no loss of $T$. colubriformis adult worms, and that we have observed similar in vitro damage on $T$. colubriformis (unpublished work) to that reported by us on other nematodes, lack of direct and prolonged access to the surface cuticle of $T$. colubriformis is the most likely explanation for the failure of PLS to show anthelmintic activity against this mucosal burrowing nematode. By tunnelling through the mucosa $T$. colubriformis may escape exposure to the direct effects of the cysteine proteinases as these pass down the gut.

The anthelmintic mechanism of action of papaya latex cysteine proteinases has been documented both in vitro and in vivo in rodents. The enzymes attack as-yet unknown protein targets in the cuticle, causing weakening of the cuticle, blistering and rupture, the release of internal tissues leading to the death of the worm. This property is not limited to the enzymes from papaya but appears to be a common property of papain homologues from pineapple, fig and from the latices of other plants [9-11,34-37].

There remains an urgent need to develop novel anthelmintics for controlling GI nematodes of domestic animals, as the available agents become less effective in the face of rapidly developing resistance by the parasites, and livestock agriculture suffers economic stresses as a consequence. We have suggested earlier [38] that resistance to naturally occurring cysteine proteinases may be very slow to develop, because the enzymes most likely target several of the constituent proteins that maintain cuticular structure and function, and therefore resistance to cysteine proteinases would be polygenic, rather than based on single point mutations as is sometimes the case for resistance to synthetic anthelmintics.

Although T. colubriformis proved to be unaffected by PLS in the current experiments, many other species of GI nematodes expose sufficient surface in the lumen of the gut to enable contact between the parasites and the enzymes. We predict that other, commercially important ruminant nematode species that reside in the gut lumen will prove to be susceptible to the action of papaya latex cysteine proteinases, thus endowing these enzymes with broad-spectrum activity.

\section{Acknowledgements}

Our work was supported by an initial grant from the University of Nottingham for a pilot trial, support from the Moredun Institute for some of the costs involved, and by the Wellcome Trust which funded both of the two experiments reported in this paper (ref no. 086479/B/08/Z). We thank Michal Chrustek, Fadlul Mansur and Wenceslaus Luoga for technical assistance.

\section{Author details}

'Department of Infection \& Immunity, University of Sheffield Medical School, Sheffield, S10 2RX, UK. ${ }^{2}$ School of Biology, University of Nottingham, University Park, Nottingham, NG7 2RD, UK. ${ }^{3}$ Parasitology Division, Moredun Research Institute, Pentlands Science Park, Bush Loan, Penicuik, Midlothian, EH26 OPZ, UK. "School of Veterinary Medicine and Science, University of Nottingham, Sutton Bonington Campus, Leicestershire, LE12 5RD, UK. ${ }^{5}$ School of Pharmacy, University of Nottingham, University Park, Nottingham, NG7 2RD, UK. 


\section{Authors' contributions}

The work was conceived by DJBut, IRD, DJBart, HME, MCG, FJ and JMB. The experiments were conducted at the Moredun Institute with the animal trials under the direct supervision of FJ, DJBart, and YB, but all authors contributed to this phase in some degree. Partial purification and characterization of the proteinase preparations was carried out by DJBut and AL. The statistical analysis was conducted by JMB. The manuscript was written by JMB and DJBut and refined by all the remaining authors. All read and approved this final version of the ms.

\section{Competing interests}

The authors declare that they have no competing interests.

Received: 3 February 2011 Accepted: 15 March 2011

Published: 15 March 2011

\section{References}

1. Kaminsky R, Ducray P, Jung M, Clover R, Rufener L, Bouvier J, Weber SS, Wieland-Berghausen S, Goebel T, Gauvry N, Pautrat F, Skripsky T, Froelich O, Komoin-Oka C, Westlund B, Sluder A, Mäser P: A new class of anthelmintics effective against drug-resistant nematodes. Nature 2008, 452:176-181.

2. Little PR, Hodges A, Watson TG, Seed JA, Maeder SJ: Field efficacy and safety of an oral formulation of the novel combination anthelmintic, derquantel-abamectin, in sheep in New Zealand. N Z Vet J 2010, 58:121-129.

3. Leathwick DM, Hosking BC: Managing anthelmintic resistance: modelling strategic use of a new anthelmintic class to slow the development of resistance to existing classes. N Z Vet J 2009, 57:203-207.

4. Besier B: New anthelmintics for livestock: the time is right. Trends Parasitol 2006, 23:21-24.

5. Hoste H, Jackson F, Athanasiadou S, Thamsborg SM, Hoskin SO: The effects of tannin-rich plants on parasitic nematodes in ruminants. Trends Parasitol 2006, 22:253-261.

6. Gradé JT, Arble BL, Weladji RB, van Damme P: Anthelmintic efficacy and dose determination of Albizia anthelmintica against gastrointestinal nematodes in naturally infected Ugandan sheep. Vet Parasitol 2008, 157:267-274

7. Behnke JM, Buttle DJ, Stepek G, Lowe A, Duce IR: Developing novel anthelmintics from plant cysteine proteinases. Parasites \& Vectors 2008, $1(29)$.

8. Dekeyser PM, Buttle DJ, Devreese B, van Beeuman J, Demeester J, Lauwers A: Kinetic constants for the hydrolysis of aggrecan by the papaya proteinases and their relevance for chemonucleolysis. Arch Biochem Biophys 1995, 320:375-379.

9. Stepek G, Lowe AE, Buttle DJ, Duce IR, Behnke JM: Anthelmintic action of plant cysteine proteinases against the rodent stomach nematode, Protospirura muricola, in vitro and in vivo. Parasitology 2007, 134:103-112.

10. Stepek $G$, Lowe $A E$, Buttle DJ, Duce IR, Behnke JM: In vitro and in vivo anthelmintic efficacy of plant cysteine proteinases against the rodent gastrointestinal nematode, Trichuris muris. Parasitology 2006, 132:681-689.

11. Stepek G, Lowe AE, Buttle DJ, Duce IR, Behnke JM: The anthelmintic efficacy of plant-derived cysteine proteinases against the rodent gastrointestinal nematode, Heligmosomoides polygyrus, in vivo. Parasitology 2007, 134:1409-1419.

12. Satrija F, Nansen P, Bjorn H, Murtini S, He S: Effect of papaya latex against Ascaris suum in naturally infected pigs. J Helminthol 1994, 68:343-346.

13. Hansson A, Veliz G, Naquira C, Amren M, Arroyo M, Arevalo G: Preclinical and clinical studies with latex from Ficus glabrata HBK, a traditional intestinal anthelminthic in the Amazonian area. J Ethnopharmacol 1986, 17:105-138.

14. Vercruysse J, Rew RS: Macrocyclic lactones in antiparasitic therapy. CABI Publishing; 2002.

15. Satrija F, Ridwan Y, Tiuria R, Retnani EB: Dampak pemberian getah papaya dengan dosis berulang terhadap domba yang diinfeksi Haemonchus contortus. Hemera Zoa 1999, 81:9-15.

16. Satrija F, Retnani EB, Ridwan Y, Tiuria R: Potential use of herbal anthelmintics as alternative antiparasitic drugs for small holder farms in developing countries. Livestock Community and Environment. Proc 10th Conference of the Assoc Institutions for Tropical Vet Med Copenhagen; 2001.
17. Barger IA, Cox HW: Wool production of sheep chronically infected with Haemonchus contortus. Vet Parasitol 1984, 15:169-175.

18. Roos MH, Boersema JH, Borgsteede FHM, Cornelissen J, Taylor M, Ruitenberg EJ: Molecular analysis of selection for benzimidazole resistance in the sheep parasite Haemonchus contortus. Mol Biochem Parasitol 1990, 43:77-88.

19. Zucker S, Buttle DJ, Nicklin MJH, Barrett AJ: Proteolytic activities of papain, chymopapain and papaya proteinase III. Biochim Biophys Acta 1985, 828:196-204.

20. Jackson F: New technique for obtaining nematode ova from sheep faeces. Lab Pract 1974, 23:65-66.

21. Palmer DG, McCombe IL: Lectin staining of trichostrongylid nematode eggs of sheep: rapid identification of Haemonchus contortus eggs with peanut agglutinin. Int I Parasitol 1996, 26:447-450.

22. Colditz IG, Le Jambre LF, Hosse R: Use of lectin binding characteristics to identify gastrointestinal parasite eggs in faeces. Vet Parasitol 2002, 105:219-222.

23. Patterson DM, Jackson F, Huntley JF, Stevenson LM, Jones DG, Jackson E, et al: Studies on caprine responsiveness to nematodiasis: segregation of male goats into responders and non-responders. Int J Parasitol 1996, 26:187-194.

24. Ministry of Agriculture FaF: Manual of veterinary parasitology laboratory techniques. Her Majesty's Stationary Office, London; 3 1986, Ref Type: Pamphlet.

25. Maley SW, Buxton D, Rae AG, Wright SE, Schock A, Bartley PM, et al: The pathogenesis of neosporosis in pregnant cattle: inoculation at midgestation. J Comp Pathol 2003, 129:186-195.

26. Barnard C, Gilbert F, McGregor P: Asking Questions in Biology. Prentice Hall, London; 2007

27. Meddis R: Statistics Using Ranks. A Unified Approach. New York: Basil Blackwell Publishers Ltd; 1984.

28. Salih E, Malthouse JPG, Kowlessur D, Jarvis M, O'Driscoll M, Brocklehurst K: Differences in the chemical and catalytic characteristics of two crystallographically 'identical' enzyme catalytic sites. Characterization of actinidin and papain by a combination of $\mathrm{pH}$-dependent substrate catalysis kinetics and reactivity probe studies targeted on the catalyticsite thiol group and its immediate environment. Biochem J 1987, 247:181-193.

29. Huet J, Looze Y, Bartik K, Raussens V, Wintjens R, Boussard P: Structural characterization of the papaya cysteine proteinases at low pH. Biochem Biophys Res Commun 2006, 341:620-626.

30. Hall CA, Oddy VH: Effect of cimetidine on abomasal pH and Haemonchus and Ostertagia species in sheep. Res Vet Sci 1984, 36:316-319.

31. Varughese KI, Ahmed FR, Carey PR, Hasnain S, Huber CP, Storer AC: Crystal structure of a papain-E-64 complex. Biochemistry 1989, 28:1330-1332.

32. Scott I, Khalaf S, Simcock DC, Knight CG, Reynolds GW, Pomroy WE, Simpson HV: A sequential study of the pathology associated with the infection of sheep with adult and larval Ostertagia circumcincta. Vet Parasitol 2000, 89:79-94.

33. Simcock DC, Scott I, Przemeck SMC, Simpson HV: Abomasal contents of parasitised sheep contain an inhibitor of gastrin secretion in vitro. Res Vet Sci 2006, 81:225-230.

34. Stepek G, Buttle DJ, Duce IR, Lowe A, Behnke JM: Assessment of the anthelmintic effect of natural plant cysteine proteinases against the gastrointestinal nematode Heligmosomoides polygyrus, in vitro. Parasitology 2005, 130:203-211.

35. Stepek G, Lowe AE, Buttle DJ, Duce IR, Behnke JM: In vitro anthelmintic effects of cysteine proteinases from plants against intestinal helminths of rodents. J Helminthol 2007, 81:353-360.

36. Berger J, Asenjo CF: Anthelmintic activity of fresh pineapple juice. Science 1939, 90:299-300.

37. Robbins BH: A proteolytic enzyme in ficin, the anthelmintic principle of leche de higueron. J Biol Chem 1930, 87:251-257.

38. Stepek G, Behnke JM, Buttle DJ, Duce IR: Natural plant cysteine proteinases as anthelmintics? Trends in Parasitol 2004, 20:322-327.

doi:10.1186/1756-3305-4-36

Cite this article as: Buttle et al: Oral dosing with papaya latex is an effective anthelmintic treatment for sheep infected with Haemonchus contortus. Parasites \& Vectors 2011 4:36. 